DOI: $10.7242 / 2658-705 X / 2019.3 .2$

УДК 621.791

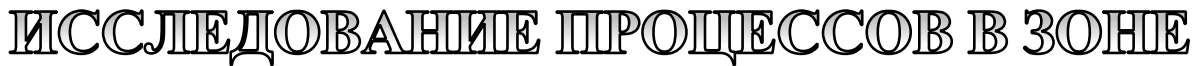

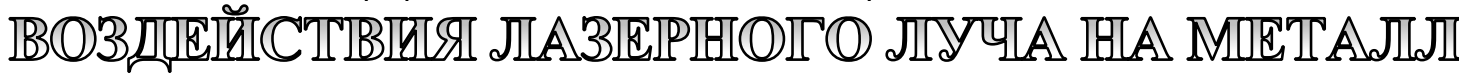

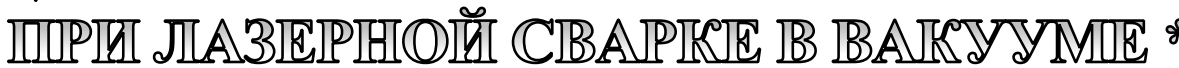

И.Ю. Летягин, Пермский национальный исследовательский политехнический университет В.Я. Беленький, Пермский наииональный исследовательский политехнический университет; Пермский военный институт войск Национальной гвардии Российской Федерации

Я.В. Лямин, Пермский национальный исследовательский политехнический университет

А.П. Ериков, Пермский военный институт войск Национальной гвардии Российской Федераџии

Приведены результаты исследований вторично-эмиссионных сигналов в зоне взаимодействия лазерного луча с металлом с целью изучения возможности осуществления контроля процесса формирования сварного шва при лазерной сварке в вакууме. Проведены экспериментальные исследования параметров вторично-эмиссионного электронного и ионного токов при импульсной лазерной сварке в вакууме. Исследованы зависимости параметров вторично-эмиссионных сигналов из зоны лазерной сварки в вакууме от расстояния между коллектором заряженных частиц, регистрирующим эти сигналы, и свариваемым изделием, а также влияние степени вакуумирования технологической камеры на параметры вторично-эмиссионных сигналов. Результаты проведенных исследований будут положены в основу разработки методов вторично-эмиссионного контроля процесса фрормирования шва при лазерной сварке в вакууме, внедрение которых в промышленное производство обеспечит высокую воспроизводимость качества сварных соединений, полученных лазерной сваркой в вакууме, и отсутствие дефектов в сварных швах.

Ключевые слова: лазерная сварка в вакууме, вторично-эмиссионный ток, контроль прочесса формирования сварного шва.

Лазерная сварка широко применяется в аэрокосмической и других высокотехнологичных отраслях промышленности для получения сварных соединений деталей из различных сталей и сплавов.

В последнее десятилетие внедрению лазерной сварки в промышленное производство способствовало появление мощных оптоволоконных лазеров, обеспечивающих высокое оптическое качество излучения.

Существенным недостатком лазерной сварки при атмосферном давлении являются потери мощности луча в плазменном факеле, образующимся над зоной воздействия мощного лазерного луча на металл. При этом лазерная сварка в атмосфере при соединении толстостенных деталей не

\footnotetext{
* Статья подготовлена при финансовой поддержке Правительства Пермского края в рамках научного проекта № C-26/794 «Лазерная сварка в вакууме - будущее сварочных технологий» и при финансировании гранта РФФИ № 16-48-590208 «Исследование взаимосвязи параметров вторично эмиссионных сигналов с процессами в парогазовом канале при лазерной сварке в вакууме».
} 
может конкурировать с электронно-лучевой сваркой, до настоящего времени широко применяющейся при изготовлении изделий ответственного назначения, что сдерживает широкое внедрение лазерной сварки в промышленное производство.

Для снижения экранирующего влияния плазменного облака и, соответственно, увеличения глубины проплавления при лазерной сварке применяются различные технологические приемы [4-7], однако они не решают проблемы, связанные с потерями мощности лазерного луча в плазменном облаке над зоной сварки.

В последние годы в высокотехнологичном сварочном производстве возрастает интерес к лазерной сварке в вакууме, которая, по сравнению с лазерной сваркой в атмосфере защитных газов, позволяет достичь значительно большей глубины проплавления при той же мощности лазерного луча $[2,8,15]$.

Ввиду того, что при формировании узких и глубоких сварных швов получение бездефектных сварных соединений при воздействии на процесс сварки различного рода возмущений возможно только при стабильном термическом воздействии лазерного луча на свариваемый металл, одним из важных вопросов является осуществление оперативного контроля процесса формирования шва.

Контроль воздействия мощных концентрированных источников энергии на металл в вакууме может осуществляться по параметрам вторичных процессов в зоне энерговыделения (эмиссии заряженных частиц, электромагнитных излучений). В частности, при сварке концентрированным электронным пучком для контроля процесса осуществляется регистрация параметров вторично-эмиссионных сигналов из зоны сварки $[1,3,19]$.

В зоне лазерной сварки в вакууме протекают процессы, аналогичные процессам при сварке мощным электронным пучком: интенсивная термоэлектронная эмиссия из конденсированной фазы металла, образование плазменного облака над зоной сварки и наличие широкого спектра колеба- тельных процессов в канале проплавления, образованном мощным концентрированным лучом лазера $[11,16,17]$.

Целью данной работы являлось изучение взаимосвязи параметров вторичноэмиссионных процессов в низкотемпературной плазме, образующейся в зоне воздействия лазерного луча на металл, с технологическими параметрами при лазерной сварке в вакууме.

Регистрация вторично-эмиссионного тока в плазме в зоне лазерной сварки в вакууме осуществлялась путем установки над зоной сварки коллектора заряженных частиц (рис. 1), включенного в электрическую цепь, содержащую резистор нагрузки и источник напряжения смещения. Сигнал с резистора нагрузки подавался на компьютерную информационноизмерительную систему с аналого-цифровым интерфейсом.

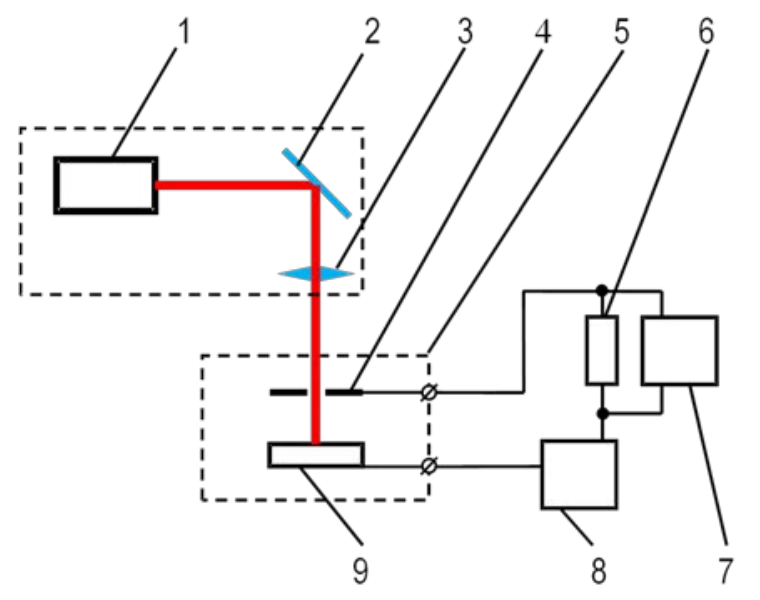

Рис. 1. Схема регистрации вторичноэмиссионного тока при лазерной сварке: 1 - лазер; 2 - отклоняюшая система; 3 - фокусирующая система; 4-коллектор заряженных частии; 5-вакуумная камера; 6 - резистор нагрузки; 7 - компьютерная информационно-измерительная система; 8 - источник напряжения смещения; 9 - свариваемое изделие

Эксперименты проводились на установке для лазерной сварки модели ALFA-300, дополненной вакуумной камерой с варьированием максимального напряжения накопителя от 200 В до 400 В, длительностью импульсов от 4 до 20 мс и частотой их следования 1 Гц. 
Экспериментальные исследования показали, что в спектре регистрируемого сигнала присутствует высокочастотная составляющая (f > 10 кГц) и ее гармоники (рис. 2)

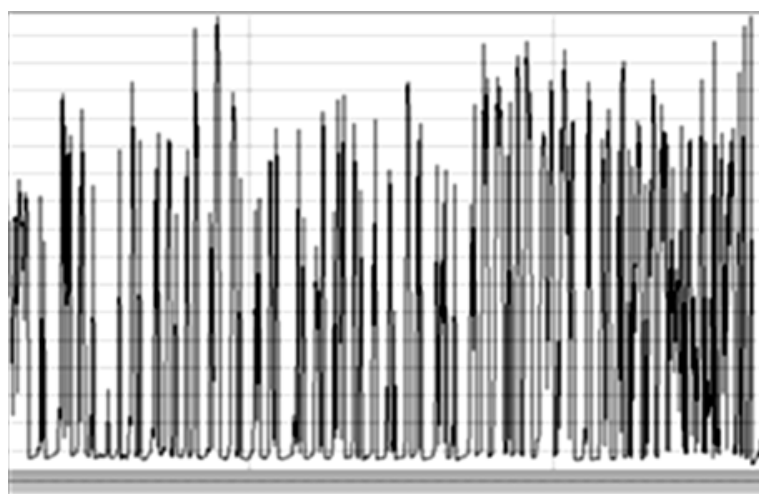

Рис. 2. Фрагмент осииллограммь вторично-эмиссионного тока в плазме, регистрируемого коллектором заряженных частии при лазерной сварке в вакууме

Эта составляющая вторично-эмиссионного тока в плазме, измеряемого с помощью коллектора заряженных частиц, отражает автоколебательные процессы в столбе плазмы при подаче на коллектор положительного потенциала. Величина сигнала возрастает при увеличении вакуума.

Природа этих автоколебательных процессов связана с возникновением ионнозвуковой неустойчивости. Наблюдаемая неустойчивость аналогична потенциально-релаксационной неустойчивости, характеризующейся большой амплитудой колебаний на положительном электроде, расположенном в плазме. Оба вида неустойчивости имеют схожую природу возбуждения и распространения. Данные неустойчивости в плазме появляются при превышении плотности тока разряда некоторой критической величины.

Расчеты показали, что при температуре ионов над зоной сварки около $2400 \mathrm{~K}$, температуре электронов $\sim 10000$ К и плотности плазмы $n_{e} \approx 10^{16} \mathrm{M}^{-3}$ критическое значение плотности тока составляет $\sim 3 \mathrm{MA} / \mathrm{M}^{2}$, что соответствует току коллектора заряженных частиц около 4 мА при его площади порядка $2 \ldots 4 \mathrm{~cm}^{2}$. Наблюдаемые в экспериментах импульсы электронного тока в плазме имеют величину, превышающую пороговое значение.
Возникающие автоколебания в плазме над зоной сварки модулируют осцилляции в спектре вторично-эмиссионного сигнала в диапазоне 100 Гц - 10 кГц, вызванные капиллярными неустойчивостями канала проплавления, стохастическим перемещением зоны взаимодействия лазерного луча с металлом на стенках канала проплавления, локальными перегревами в канале проплавления, пульсациями потоков паров из канала проплавления и другими периодическими процессами в канале проплавления.

В зоне лазерной сварки при атмосферном давлении, вследствие известного в физике плазмы механизма столкновительного затухания колебаний, при высоких концентрациях нейтральных атомов возрастает вероятность соударений частиц плазмы за период колебаний. Число этих столкновений достаточно велико, и колебания затухают очень быстро.

Была поставлена задача дифференцировать колебательные процессы в плазме, связанные с различного вида неустойчивостями, и автоколебания, вызываемые периодическими процессами в канале проплавления, так как последние должны в большей степени коррелировать с технологическими параметрами лазерной сварки в вакууме и с геометрией зоны проплавления в металле при воздействии лазерного луча.

Ввиду того, что характер автоколебаний в плазме, связанных с различного рода неустойчивостями, в значительной степени определяется геометрией плазменного облака, образующегося в зоне лазерной сварки в вакууме, были проведены исследования влияния расстояния между коллектором заряженных частиц, осуществляющим отбор вторичного тока из плазмы, и поверхностью металла, подвергаемого воздействию лазерного луча, на амплитудно-временные параметры вторично-эмиссионного сигнала.

На рис. 3 представлены спектры колебаний, полученные при математической обработке в среде MathCad результатов записи вторично-эмиссионных сигналов в зоне лазерной сварки в вакууме с исполь- 


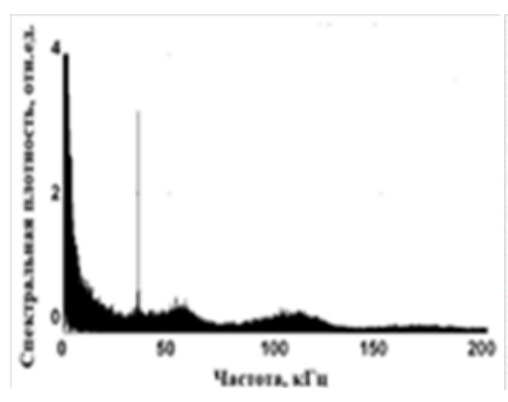

$a$

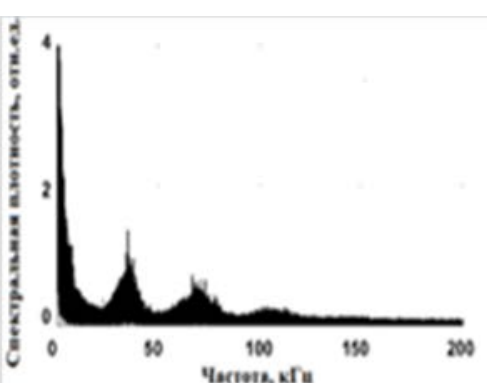

$\sigma$

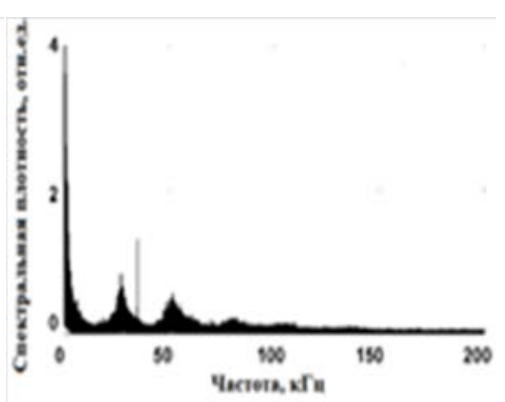

$\sigma$

Рис. 3. Спектры колебаний вторично-эмиссионного тока в плазме, образующейся в зоне лазерной сварке в вакууме, при различных расстояниях $Z$ от коллектора заряженньх частич до поверхности металла ( $а-10$ мм, б-20 мм, в - 30 мм)

зованием компьютерной информационноизмерительной системы при различных расстояниях от коллектора заряженных частиц до поверхности металла.

На рис. 4 приведена зависимость частоты компоненты спектра вторично-эмиссионного тока, обусловленной ионно-звуковыми колебаниями в плазме в зоне лазерной сварки в вакууме, от расстояния между колектором заряженных частиц и поверхностью металла

Как видно из рис. 3 и 4, при уменьшении расстояния от поверхности металла до коллектора заряженных частиц спектральная плотность высокочастотных компонент спектра вторично-эмиссионного тока в зоне лазерной сварки в вакууме, связанных с ионно-звуковой и релаксационной неустойчивостями в плазме, уменьшается, и увеличивается их частота. Это свидетельствует о том, что при регистрации вторичноэмиссионного тока с целью контроля геометрических параметров проплавления при лазерной сварке в вакууме необходимо размещать электрод-коллектор заряженных частиц в непосредственной близости к поверхности свариваемого металла.

Следующим этапом исследований вторично-эмиссионных процессов при лазерной сварке в вакууме было изучение влияния степени вакуумирования технологической камеры установки для лазерной сварки в вакууме на амплитудно-временные параметры вторично-эмиссионного тока. При проведении этих исследований вакуум в технологической камере изменяли дросселированием потока откачиваемого возду- ха путем регулирования проходного сечения трубопровода, соединяющего технологическую камеру с вакуумным насосом.

На рис. 5 приведены осциллограммы вторично-эмиссионного тока в плазме в зоне лазерной сварки в вакууме при различном давлении в технологической вакуумной камере. Результаты экспериментов показали, что при повышении давления в

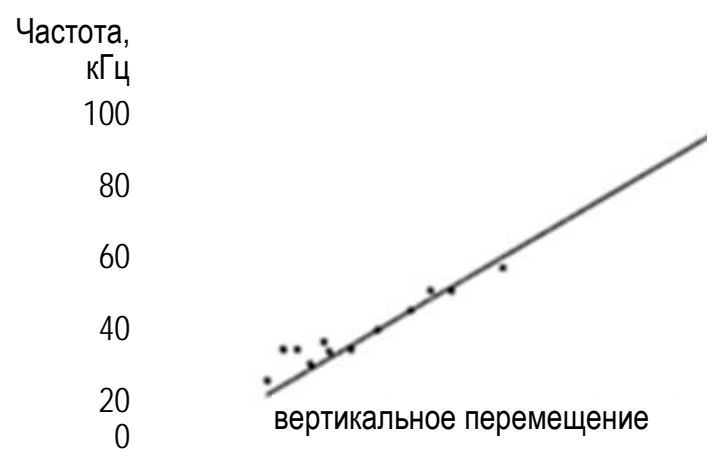

$\begin{array}{lllllll}0 & 10 & 20 & 30 & 40 & 50 & 1 / \mathrm{R}, \mathrm{M}^{-1}\end{array}$ Частота, КГц 60 50 40 40 30 20 10 $\begin{array}{llllllll}0 & 10 & 20 & 30 & 40 & 50 & 60 & 1 / R, M^{-1}\end{array}$

Рис.4. Зависимость частоты компоненты спектра, обусловленной ионно-звуковыми колебаниями в плазме в зоне лазерной сварки в вакууме от расстояния между колектором заряженных частиц и поверхностью металла 

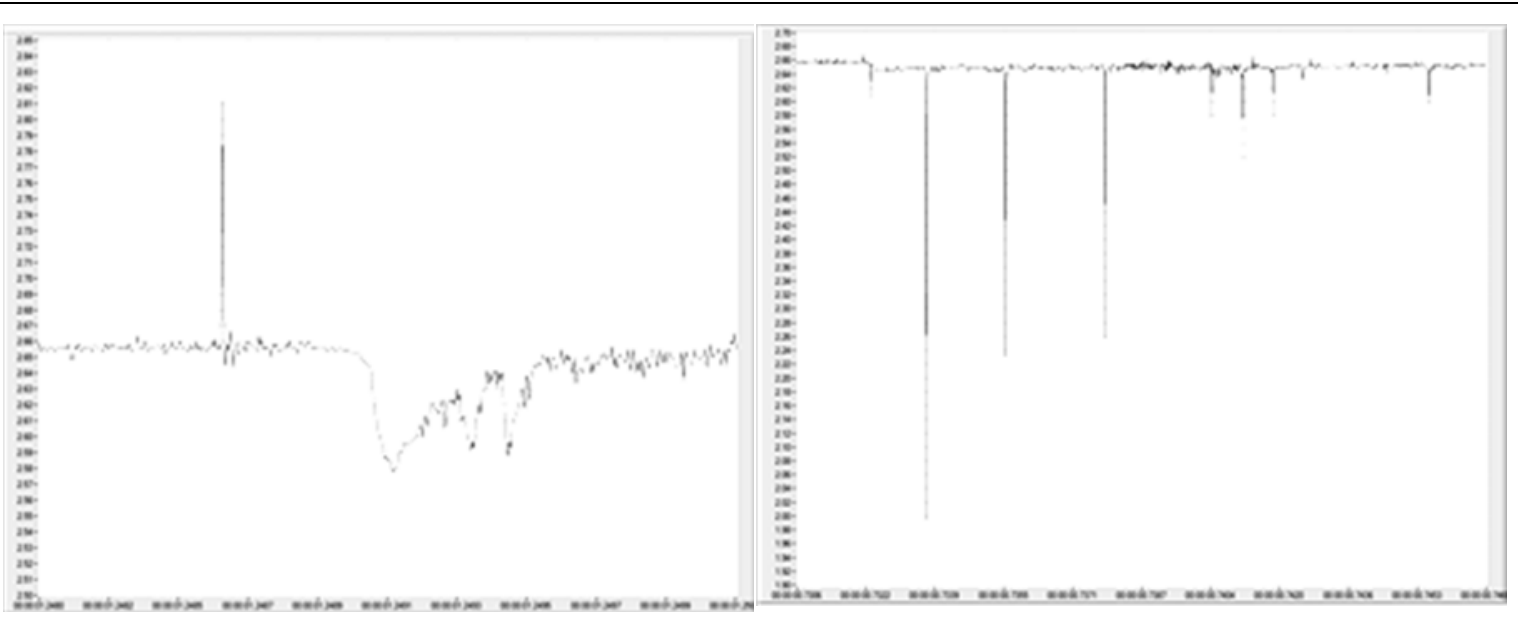

$a$

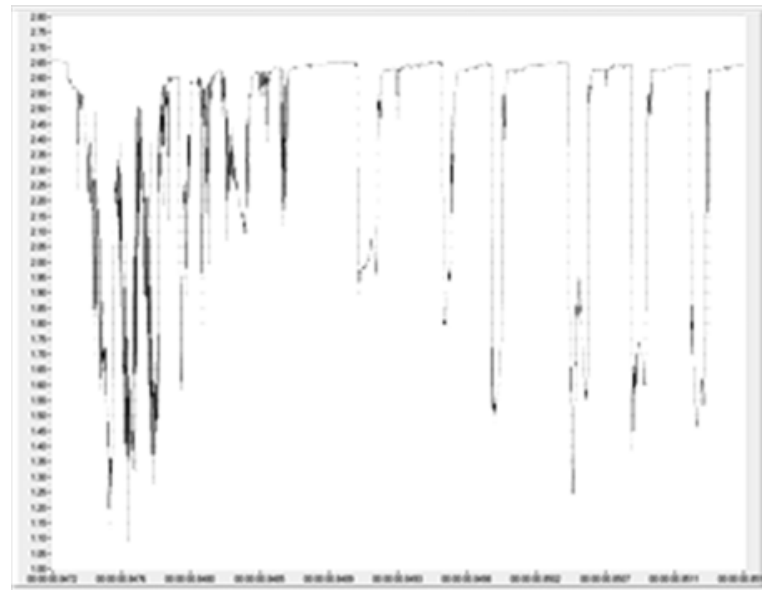

Рис. 5. Осцииллограммы вторично-эмиссионного тока давлении в технологической вакуумной камере: $a-4000 \Pi а, \sigma-100 \Pi а$, в - 10 Па

технологической вакуумной камере установки для лазерной сварки в вакууме амплитуда импульсов вторично-эмиссионного тока в плазме уменьшается и становится равной нулю при давлении в камере, близком к атмосферному. Применительно к колебательным процессам в плазме, возникающим в результате различного вида неустойчивостей, это связано с тем, то в зоне лазерной сварки при давлении в технологической камере, близком к атмосферному, вследствие известного в физике плазмы механизма столкновительного затухания колебаний, при высоких концентрациях нейтральных атомов возрастает вероятность соударений частиц плазмы за период колебаний. Число этих столкновений при давлении, близком к атмосферному, достаточно велико, и колебания зату- хают очень быстро. Что касается колебаний вторично-эмиссионного тока в плазме, обусловленных периодическими процессами в зоне воздействия концентрированного лазерного луча на металл, то существенное снижение их амплитуды вызвано уменьшением проводимости плазмы в результате уменьшения длины свободного пробега носителей заряда и снижения скорости их дрейфа в плазме.

Проведено численное моделирование формирования электронного и ионного тока в плазме при лазерной сварке в вакууме. В основе разработанной модели лежат уравнения переноса для концентрации электронов и средней энергии электронов в плазме над зоной сварки.

Для описания массопереноса тяжелых частиц плазмы (ионов, нейтральных невоз- 
бужденных и возбужденных атомов) используется уравнение переноса массы для многокомпонентной смеси. Электрическое поле определялось из уравнения Пуассона.

Численная реализация модели осуществлялась в осесимметричной цилиндрической постановке. Для получения численного решения использовался пакет прикладного программного обеспечения COMSOL 4.4 модули Plasma Module. Образование плазмы рассматривалось как результат ионизации истекающих из канала проплавления паров металла лазерным лучом. Плотность смеси задается уравнением состояния идеального газа.

Так как моделирование было направлено, в первую очередь, на понимание физических процессов, протекающих при регистрации сигналов вторичных токов в плазме, в качестве приближения была использована линейная аппроксимация известных из литературы данных. Значения давления и температуры на дне канала проплавления приняты 7 кПа и $2700 \mathrm{~K}$ соответственно, в верхней части - 3 кПа и 2200 К. Над зоной сварки течение постепенно переходит в свободномолекулярное. Распределение источника ионизации принималось цилиндрическим, действующим вдоль распространения лазерного луча. Мощность источника калибровалась с целью максимального удовлетворения результатов моделирования распределения параметров плазмы в отсутствии положительного коллектора экспериментальным данным и данным, известным в литературе.

Важной особенностью модели являлось то, что она позволяет рассчитать параметры плазмы не только над зоной сварки, но и непосредственно в формирующемся парогазовом канале.

На рис. 6 приведено распределение потенциала в плазме над зоной сварки и в канале проплавления при регистрации вторично-эмиссионного тока коллектором заряженных частиц, находящимся под положительным потенциалом.

Разработанная модель позволяет рассчитать изменение параметров при возбу-

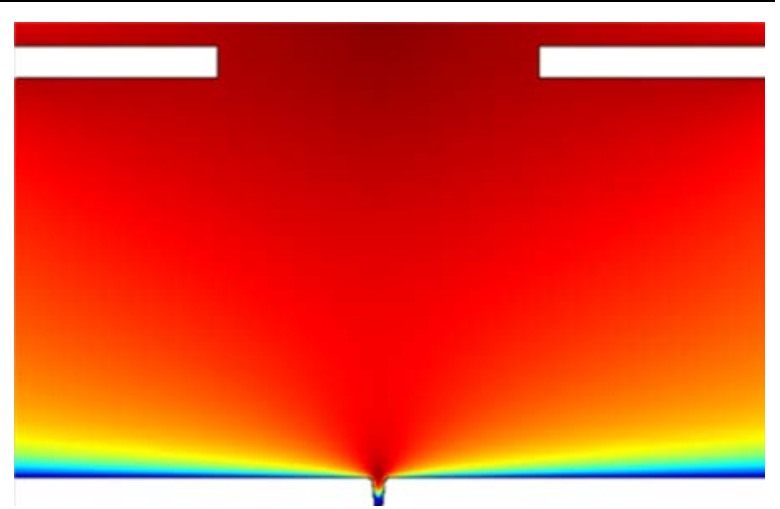

Рис. 6. Распределение потенцииала в плазме над зоной сварки и в канале проплавления при возбуждении несамостоятельного разряда ждении несамостоятельного разряда путем подачи на коллектор положительного потенциала. В первую очередь изменения заметны в распределении потенциала. Потенциал практически во всей области становится близок к установившемуся напряжению на коллекторе. Вблизи катода (свариваемого изделия) возникает слой, на котором происходит изменение этого потенциала до нуля. В канале проплавления этот слой становится особенно тонким, что приводит к возникновению у поверхности сильного электрического поля.

Расчеты показали, что по всей длине стенки канала проплавления напряженность электрического поля составляет величину порядка $10^{5} \ldots 10^{6} \mathrm{~B} / \mathrm{M}$. Столь сильное электрическое поле существенно снижает высоту потенциального барьера и работу выхода электронов. Плотность эмиссионного тока в этом случае описывается выражением Ричардсона-Шоттки. Получена зависимость вторичного тока в плазме от геометрических параметров канала проплавления. Величина вторичного тока в плазме падает с увеличением глубины проплавления по закону, близкому к экспоненциальному. Данная зависимость обусловливает экстремальный характер изменения величины вторичного сигнала в зависимости от фокусировки лазерного луча и других технологических параметров, что может быть использовано при построении методов контроля процессом. При изменении внешнего давления величина сигнала 
монотонно уменьшается по закону, близкому к обратному, что удовлетворительно согласуется с экспериментальными данными.

Была проведена верификация результатов расчета с использованием экспериментальных данных. Погрешность расчетных результатов не превышала 4-8\% от экспериментально полученных данных.

Для оценки температуры в канале проплавления, образованном в металле при лазерной сварке в вакууме, проводилось численное моделирование истечения паров из канала проплавления в зависимости от внешнего давления.

При построении модели процессов испарения при лазерной сварке в вакууме были приняты следующие предположения:

- форма канала проплавления, образуемого мощным концентрированным лазерным лучом в металле, имеет цилиндрическую форму;

- температура поверхности канала проплавления в металле во всех точках одинакова;

- тепломассоперенос вдоль оси канала проплавления не учитывается;

- скорость истечения паров металла из канала проплавления равна скорости звука.

Таким образом, задача моделирования истечения паров из канала проплавления сводилась к двумерной, при этом расчетная включает в себя и область с жидкой фазой металла.

Система уравнений, описывающая процессы испарения при лазерной сварке в вакууме, включала уравнение переноса импульса (уравнение Навье-Стокса) и уравнение переноса энергии в металле.

Система дифференциальных уравнений замыкалась краевыми условиями, включающими начальное распределение температуры и граничные условия. На входной границе задавались постоянные значения концентрации и температуры, а на выходной границе - условия постоянства плотности теплового потока. Поверхность канала проплавления в металле принималась изотермической.
Численное моделирование истечения паров металла из канала проплавления при воздействии на металл лазерного луча в вакууме производилось с использованием программного пакета Comsol Multiphysics. Одним из входных параметров модели являлась температура стенок канала проплавления в металле. Для каждого значения этого параметра из решения сопряженной системы уравнений, описывающих испарение, конденсацию и тепломассоперенос, определялись давление, поля температур и скоростей потока пара.

Для оценки температуры в канале проплавления, образованном лазерным лучом в металле при лазерной сварке в вакууме, экспериментальные данные сопоставлялись с результатами численных расчетов. При экспериментальных исследованиях производилось измерение концентрации элементов в парах над зоной взаимодействия путем химического анализа напыленного на подложку слоя металла. Значения температуры парогазовой фазы в канале проплавления определялись по равенству расчетных и экспериментальных значений концентрации элементов и составили $2150 \mathrm{~K}$ для лазерной сварки в вакууме и $2330 \mathrm{~K}$ для лазерной сварки при атмосферном давлении.

Были проведены исследования параметров ионного тока в плазме в зоне лазерной сварки в вакууме, который регистрировался при создании отрицательного потенциала на коллекторе заряженных частиц в схеме, изображенной на рис. 1.

На рис. 7 приведена осциллограмма ионного тока в плазме, образующейся при воздействии лазерного луча на образец из стали 12 Х187Н10T. Применительно к использованию вторично-эмиссионных сигналов для контроля геометрических параметров проплавления при лазерной сварке в вакууме регистрация ионного тока представляет особый интерес, так как в этом случае регистрируемые параметры сигнала не связаны с возбуждением плазменных автоколебаний и величина ионного тока непосредственно отражает колебания плотности истекающих из канала паров металла. 


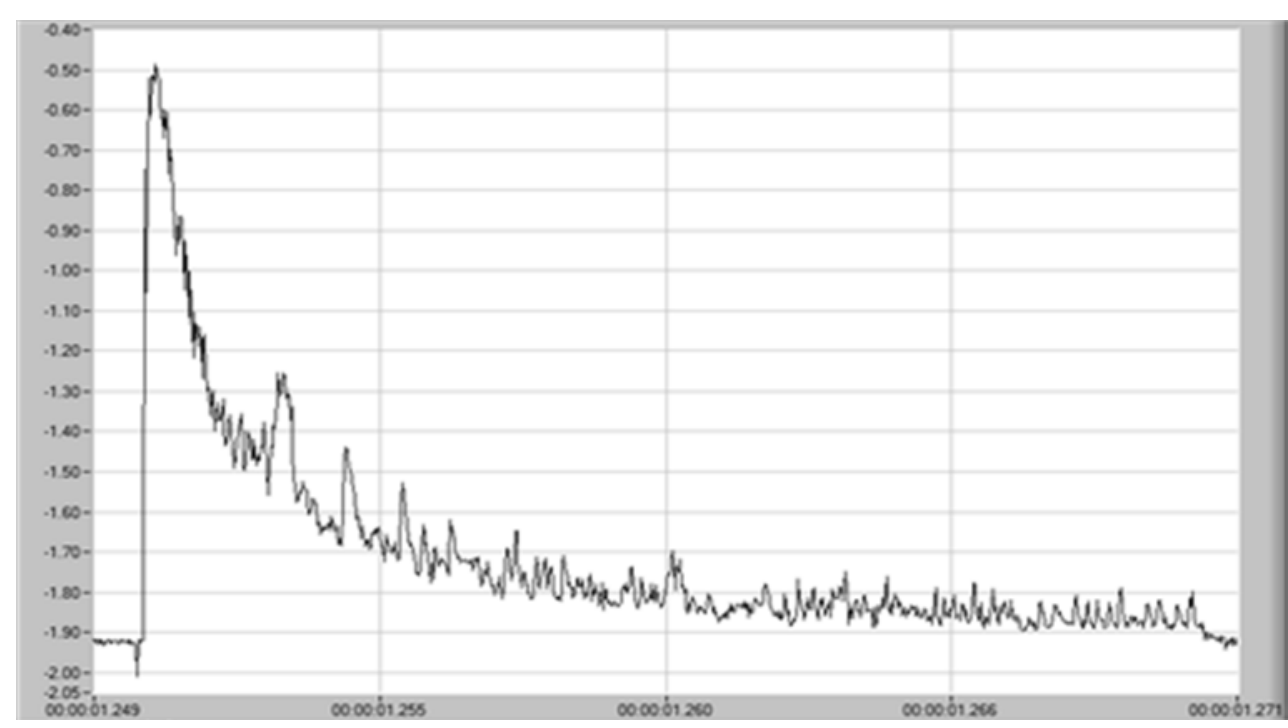

Рис. 7. Осциллограмма ионного тока в плазме, образующейся при воздействии лазерного луча на металл в вакууме

При проведении экспериментов был также произведен поиск параметров плазмы, характеризующих появление полного проплавления металла лазерным лучом. Установлено, что появление полного проплавления при небольшой глубине сварки сопровождается снижением ионного тока в плазме, измеряемого в цепи отрицательного электрода, помещенного над зоной сварки. Данный эффект объясняется тем, что регистрируемый сигнал пропорционален степени ионизации, а значит, и плотности паров металла над зоной воздействия лазерного луча, которая при появлении сквозного проплавления снижается.

Для исследования влияния переменного магнитного поля в технологическую вакуумную камеру установки для лазерной сварки в вакууме на базе сварочной установки ALFA-300 была вмонтирована электромагнитная система, представляющая собой низкоиндуктивную катушку без ферромагнитного сердечника, намотанную на фторопластовом каркасе. Эта катушка устанавливалась вблизи зоны взаимодействия лазерного луча с металлом в вакууме и подключалась к выходу широкополосного усилителя мощности, на вход которого подавался сигнал переменного тока синусоидальной формы от генератора сигналов. В остальном схема эксперимента соответствовала схеме, приведенной на рис. 1. Частоту тока в катушке изменяли в диапазоне $10 \ldots 50$ кГц, что соответствует диапазону частот регистрируемых колебаний вторично-эмиссионных сигналов в плазме при лазерной сварке в вакууме.

В результате экспериментов было установлено, что существуют резонансные режимы электромагнитного воздействия, при которых наблюдается значительное увеличение амплитуды колебаний вторичного электронного тока. Было установлено, что в спектре наблюдается максимум на резонансной частоте воздействия. Однако при анализе геометрических параметров зон проплавления в металле при лазерной сварке в вакууме в исследованном диапазоне технологических параметров существенных различий геометрии зон проплавления при резонансном воздействии на плазму и при отсутствии резонанса обнаружено не было.

Результаты проведенных экспериментальных исследований параметров вторично-эмиссионных процессов при импульсной лазерной сварке в вакууме будут положены в основу разработки методов оперативного контроля процесса формирования шва при лазерной сварке в вакууме. Эти методы обеспечат разработку бездефектных технологий лазерной сварки в вакууме, которые будут внедрены на машиностроительных предприятиях и предприятия х аэрокосмической отрасли Пермского края. 


\section{Библиографический список}

1. Беленький В.Я., Трушников Д.Н. Исследование формирования сигнала вторичного тока в плазме при электронно-лучевой сварке с осцилляцией электронного пучка // Сварочное производство. - 2012. № 11. - С. 9-13.

2. Беленький В.Я., Трушников Д.Н., Летягин И.Ю. [и др.] Лазерная сварка в вакууме - перспективная сварочная технология изготовления изделий ответственного назначения // Вестник Пермского национального исследовательского политехнического университета. Машиностроение, материаловедение. - 2014. - Т. 16. Вып. 4. С. 71-81.

3. Бойко B.A. [и др.] Рентгеновская спектроскопия лазерной плазмы // В кн.: Итоги науки и техники. Радиотехника. - Т. 27. - М., 1980.

4. Веденов А.А., Гладуш Г.Г. Физические процессы при лазерной обработке материалов. - М.: Энергоатомиздат, 1985. - $208 \mathrm{c}$.

5. Григорьяни А.Г. Основы лазерной обработки материалов. - М.: Машиностроение, 1989. - 304 с.

6. Виноградов Б.А., Гавриленко В.Н., Либенсон М.Н. Теоретические основы воздействия лазерного излучения на материалы. - Благовещенск: изд. БПИ, 1993. - 344 с.

7. Зуев И.В. Обработка материалов концентрированными потоками энергии. - М.: Изд-во МЭИ, 1997. $437 \mathrm{c}$.

8. Летягин И.Ю. Технологические перспективы лазерной сварки в вакууме // Материалы междунар. НТК «Сварка и родственные технологии». - Екатеринбург, 2016. С. 6-7.

9. Летягин И.Ю., Федосеева Е.М. Оценка сквозного проплавления при лазерной сварке на основе регистрации плазменного факела // Вестник Пермского национального исследовательского политехнического университета. Машиностроение, материаловедение. - 2016. Т. 18. Вып. 1. С. 84-100.

10. Миркин Л.И. Физические основы обработки материалов лучами лазера. - М.: Изд-во МГУ, 1975. - 383 с.

11. Основы физики лазеров и лазерной обработки материалов: учеб. пособие / Д.М. Гуреев, С.В. Ямщчиков, - Самара: Самарский университет, 2001. - 392 с.

12. Трушников Д.Н. Изучение физических процессов при электронно-лучевой сварке по параметрам вторичного тока в плазме // Физика и химия обработки материалов. - 2015. - №5. - С. 36-45.

13. Янчук Л.М. Зуев И.В., Углов А.А. О влиянии степени заглубления фокуса в материал на параметры проплавления при электронно-лучевой сварке // Сварочное производство. - 1974. - № 12. - С. 3-4.

14. Current-driven ion-acoustic and potential-relaxation instabilities excited in plasma plume during electron beam welding // Mladenov G.M., Koleva E.G., Varushkin S.V., Trushnikov D.N., Belenkiy V.Y. // AIP Advances. 2014. - Vol. 4. - Iss. 4.

15. Letyagin I.Yu., Belenkiy V.Ya., Trushnikov D.N. Benefits and Prospects of Laser Welding Application in Vacuum // IV Sino-Russian ASRTU Symposium on Advanced Materials and Processing Technology (2016): ASRTU conf. proc. / Knowledge E Engaging minds. - Dubai: Knowledge E, 2016. - (KnE Materials Science, ISSN 2519-1438; Vol. 2016). [Электронный pecypc] URL: http://knepublishing.com/index.php/KnE-Materials/article/view/568/1818 (дата обращения:15.05.2019).

16. Letyagin I.Yu., Trushnikov D.N., Belenkiy V.Ya. The studies of plasma torch processes by laser beam welding // Mechanical Engineering, Materials Science and Civil Engineering IV (4th ICMEMSCE): [sel., peer rev. papers from the 4th Intern. Conf. on Mechanical Engineering, Materials Science and Civil Engineering (ICMEMSCE 2016), November 19-20, 2016, Sanya, China] / ed. J. Zhao; Trans Tech Publications Inc. - Pfaffikon : TTP, 2017. - ([Materials Science Forum, ISSN 1662-9752 ; Vol. 893]).

17. Lieberman M.A., Lichtenberg A.J. Principles of plasma discharges and materials processing // MRS Bulletin. - 1994. - Vol. 30. - P. 899-901.

18. Trushnikov D.N., Belenkiy V.Ya.,.M., Portnov N.S. Secondary - Emission signal for weld formation monitoring and control in electron beam welding (EBW) // Materialwissenschaft und Werkstofftechnik. 2012. - Vol. 43. - № 10. - P. 892-897.

19. Trushnikov D.N., Belenkiy V.Ya., Salomatova E.S. Formation of a Secondary Current Signal in Electron Beam welding of Dissimilar Materials // Global Journal of Pure and Applied Mathematics. - 2016, - Vol. 12, - № 1. P. 657-676. 


\title{
PROCESSES IN THE AREA OF LASER BEAM EXPOSURE TO METAL RESEARCH DURING LASER WELDING IN VACUUM
}

\author{
I.Yu. Letyagin ${ }^{1}$, V.Ya. Belenkiy ${ }^{1,2}$, Ya.V. Lyamin ${ }^{1}$, A.P. Erikov ${ }^{2}$ \\ ${ }^{1}$ Perm National Research Polytechnic University \\ ${ }^{2}$ Perm Military Institute of National Guard Troops
}

Research results are presented of secondary emission signals in the zone of interaction of a laser beam with metal in order to study the possibility of monitoring the process of formation of laser weld in vacuum. Experimental studies of the parameters of the secondary emission electron and ion currents during pulsed laser welding in vacuum have been carried out. The dependences of the parameters of the secondary emission signals from the laser welding zone in vacuum on the distance between the collector of charged particles registering these signals and the product being welded, as well as the effect of the degree of vacuuming of the process chamber on the parameters of the secondary emission signals, are investigated. The results of the research will make up the basis for the development of methods of secondary emission control of the process of weld formation during laser welding in vacuum; its implementation into industrial production will ensure high reproducibility of the quality of welded joints produced by laser welding in vacuum and the absence of defects in welds.

Keywords: laser welding in vacuum, secondary-emission current, control of the weld formation process.

\section{Сведения об авторах}

Летягин Игорь Юрьевич, кандидат технических наук, доцент кафедры «Сварочное производство, метрология и технология материалов», Пермский национальный исследовательский политехнический университет (ПНИПУ), 614990, г. Пермь, Комсомольский пр., 29; e-mail: letyagin@pstu.ru

Беленький Владимир Яковлевич, доктор технических наук, профессор кафедры «Сварочное производство, метрология и технология материалов», ПНИПУ, Пермский военный институт войск Национальной гвардии Российской Федерации (ПВИ войск Национальной гвардии), 614112, г. Пермь, ул. Гремячий Лог, д.1; e-mail: vladimirbelenkij@yandex.ru

Лямин Яков Васильевич, кандидат технических наук, доцент кафедры «Сварочное производство, метрология и технология материалов», ПНИПУ; e-mail: yakov lyamin@mail.ru

Ериков Алексей Петрович, доцент, ПВИ войск Национальной гвардии; e-mail: yerikov@yandex.ru

Материал поступил в редакцию 07.07.2019 г. 